\title{
Kynurenic Acid in Plasma and Endometrium in Bitches with Pyometra
}

\author{
Roman Dąbrowski, ${ }^{1}$ Tomasz Kocki, ${ }^{2}$ Marek Szczubial, ${ }^{1}$ Wojciech Dąbrowski, ${ }^{3}$ and \\ Jolanta Parada-Turska ${ }^{4,5}$
}

\begin{abstract}
Kynurenic acid (KYNA) is produced enzymatically in humans and animals from kynurenine. Reports concerning changes of kynurenine metabolism during inflammation are available in the literature. Pyometra is a pathological condition characterized by the accumulation of pus in the uterine lumen and bacterial infection. The objective of the study was to compare the serum and endometrial KYNA concentrations in healthy bitches and those with pyometra. KYNA was determined by means of highperformance liquid chromatography with fluorometric detection. The serum content of KYNA in bitches with pyometra was significantly higher than in healthy bitches. The KYNA content in the endometrium of bitches with pyometra was higher, yet the difference was not statistically significant. Our result indicates that determination of KYNA might be a marker of pyometra in bitches.
\end{abstract}

KEY WORDS: bitches; inflammation; kynurenic acid; pyometra.

\section{INTRODUCTION}

Kynurenic acid (KYNA) was first detected in dog's urine and described in 1853 by Liebig. It is produced in vertebrates along the kynurenine pathway of tryptophan metabolism [1]. KYNA is a final product of the kynurenine transamination [2]. Apart from its action on neuronal glutamatergic receptors [3, 4], KYNA is also a ligand of $G$ protein-coupled receptor 35 , which demonstrates a high expression on the immune system and the gastrointestinal tract components [5] as well as a ligand of aromatic hydrocarbon receptor participating in the process of carcinogenesis and mechanisms of immune

\footnotetext{
${ }^{1}$ Department and Clinic of Animal Reproduction, Faculty of Veterinary Medicine, University of Life Sciences in Lublin, Głęboka 30, 20-612 Lublin, Poland

${ }^{2}$ Department of Experimental and Clinical Pharmacology, Medical University in Lublin, Jaczewskiego 8, 20-090 Lublin, Poland

${ }^{3}$ Department of Anaesthesiology and Intensive Therapy, Medical University in Lublin, Jaczewskiego 8, 20-954 Lublin, Poland

${ }^{4}$ Department of Rheumatology and Connective Tissue Diseases, Medical University in Lublin, Jaczewskiego 8, 20-954 Lublin, Poland

${ }^{5}$ To whom correspondence should be addressed at Department of Rheumatology and Connective Tissue Diseases, Medical University in Lublin, Jaczewskiego 8, 20-954 Lublin, Poland. E-mail: jolanta.turska@am.lublin.pl
}

response modulation [6]. There are premises of KYNA's significance in various inflammatory diseases [7-17].

Pyometra is one of the most frequently occurring pathological conditions of the reproductive tract in adult and older bitches, in the metestrus phase of the estrous cycle. The disease is characterized by the accumulation of a large amount of pus in the uterine lumen, which, in consequence, leads to absorption of suppurating toxins by blood and systemic disturbances, e.g., kidney and liver damage [18].

Pyometra often constitutes a threat to the bitches' life, and, for this reason, it should be diagnosed and treated as soon as possible. The primary treatment in a case of pyometra is surgical intervention consisting of the simultaneous removal of both the uterus and ovaries [19].

So far, there are no literature data concerning the determination of KYNA in inflammation of uterus in animals. The objective of the study was to compare the serum and endometrial KYNA concentrations in healthy bitches and those with pyometra.

\section{MATERIALS AND METHODS}

All experimental protocols and procedures were approved by the First Local Ethics Committee in Lublin. 
The study involved 20 bitches of various breeds and mongrels treated in the Department and Clinic of Animal Reproduction, Veterinary Faculty, University of Life Sciences in Lublin. The animals were divided into two groups. Control group consisted of ten clinically healthy bitches, aged 2-6 years, between the 4-11th weeks after estrous cycle, which underwent ovariohysterectomy on the owners' request. Study group included ten bitches, aged 5-13 years, diagnosed with pyometra based on the medical history, clinical examination, cytology tests, vaginoscopy, and ultrasound examination as well as hematological and blood biochemistry tests. Bitches in this group were between 4 and 10 weeks after estrous cycle. These animals underwent ovariohysterectomy because of pyometra.

In both groups, the surgical procedures were carried out under general anesthesia using intramuscular ketamine of $5-15 \mathrm{mg} / \mathrm{kg}$ b.w. (Narkamon $5 \%{ }^{\circledR}$, Spofa, Czech Republic), following premedication with subcutaneous atropine of $0.05 \mathrm{mg} / \mathrm{kg} \mathrm{b}$.w. (Atropinum sulfuricum ${ }^{\circledR}$, Polfa, Poland), and intramuscular xylazine of $2 \mathrm{mg} / \mathrm{kg}$ b.w. (Rometar $2 \%{ }^{\circledR}$, Spofa, Czech Republic).

Prior to surgery, the blood was sampled $(9 \mathrm{ml})$ from the saphenous vein of all bitches to sterile centrifugation tubes and silicone vacuette (Greiner Labortechnik $\mathrm{GmbH}$, Austria). After blood centrifugation, the serum was frozen and kept at $-80{ }^{\circ} \mathrm{C}$ until used.

Immediately after surgery, the uterine endometrium biopsies were taken. The endometrium section, $1.0 \times 1.0 \mathrm{~cm}$, was excised from the central right horn of the uterus, placed in the plastic Eppendorf tubes and frozen at $-80^{\circ} \mathrm{C}$.

KYNA was investigated according to the method of Turski et al. [20] and Shibata [21]. In brief, serum samples were acidified with $1 \mathrm{~N} \mathrm{HCl}$ and $50 \%$ trichloroacetic acid and then centrifuged. Tissue samples were sonicated $(1: 5 \mathrm{wt} / \mathrm{vol})$ in distilled water. The resulting homogenate was centrifuged. The supernatant was acidified with $1 \mathrm{~N} \mathrm{HCl}$ and $50 \%$ trichloroacetic acid and then centrifuged. Supernatant was applied on cation-exchange resin (Dowex $50 \mathrm{~W}+$, Sigma). Eluted KYNA was subjected to the HPLC (Varian HPLC system, ESA catecholamine HR-80, $3 \mu \mathrm{m}, \mathrm{C}_{18}$ reversephase column) and quantified fluorometrically (excitation, $344 \mathrm{~nm}$; emission, $398 \mathrm{~nm}$ ).

The results are presented as a mean \pm standard deviation or median values according to data distribution and result of normality test. Data were analyzed using Student $t$ test or Mann-Whitney test. The significance of differences was determined at $p<0.05$.

\section{RESULTS}

All bitches with pyometra showed reduced appetite, polydipsia, apathy, and enlarged abdominal integuments; $80 \%$ of them developed purulent discharge from reproductive organs. In all bitches, abdominal ultrasound examinations revealed enlarged uterus of a diameter ranging from 2 to $7 \mathrm{~cm}$ with a hypoechogenic content.

The mean amount of leucocytes in bitches with pyometra was $27.5 \pm 8.0 \times 10^{9} / 1$ and was statistically significantly higher $(p<0.05)$ compared to the values in healthy bitches $\left(11.4 \pm 2.5 \times 10^{9} / 1\right)$. The percentage of rod neutrophils noted in bitches suffering from pyometra $(5.9 \pm$ $1.5 \%)$ was statistically significantly higher $(p<0.05)$ compared to that of healthy bitches $(1.7 \pm 0.7 \%)$.

According to the bacteriological tests of the uterine purulent secretions, Escherichia coli was identified in $90 \%$ of cases; in the remaining $10 \%$, Staphylococcus spp. were detected.

The median serum content of KYNA in bitches with pyometra and healthy bitches was 179.6 and 49.0 $\mathrm{pmol} / \mathrm{ml}$, respectively (Fig. 1). The difference was statistically significant $(p<0.05)$. The median content of KYNA in the endometrium of bitches with pyometra and in healthy animals was 334.1 and $121.5 \mathrm{pmol} / \mathrm{g}$, respectively (Fig. 2). The difference did not reach statistical significance $(p>0.05)$.

Average age of animals with pyometra was $10.8 \pm$ 2.2 years and was significantly higher $(p<0.05)$ compared to that of healthy bitches $(3.4 \pm 1.4$ years). There was no correlation between KYNA content and age in healthy bitches (data not shown).

\section{DISCUSSION}

Results of clinical examination, laboratory, and bacteriological tests confirm the diagnosis of pyometra in the investigated bitches. In bitches with pyometra, a significant increase of blood KYNA concentration has been demonstrated. Our result is consistent with other reports. An increase of KYNA in serum was reported in generalized sepsis in humans [7] and in patients with chronic kidney disease [8]. A significant increase of KYNA in patients with meningitis, septicemia and various autoimmunological diseases as well as with bacterial, viral, fungal, and parasitic infections was demonstrated in the cerebrospinal fluid and in the brain [9]. Similarly, an increase in KYNA concentration in the brain of mice infected with the flu virus has been observed 


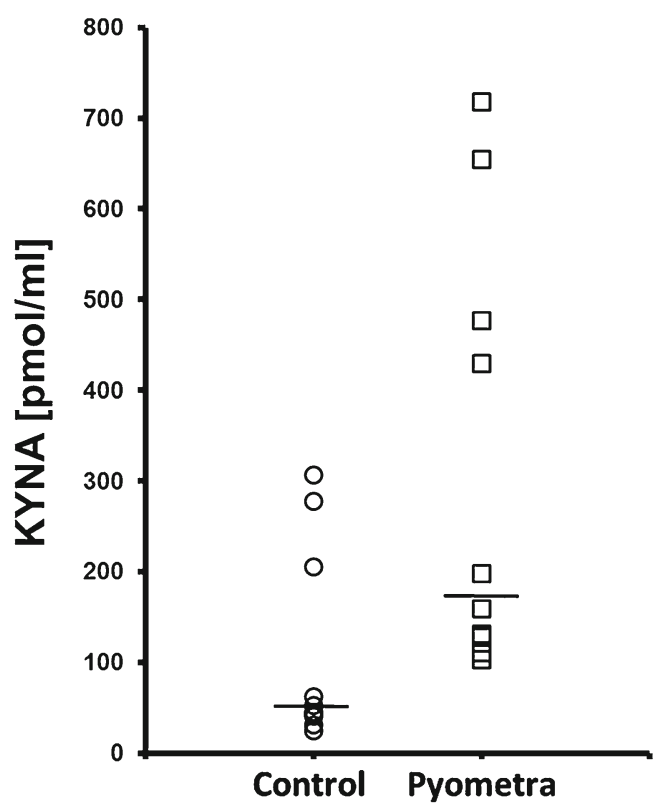

Fig. 1. Scatter plots of kynurenic acid (KYNA) concentration in plasma of bitches. Samples were collected from bitches that underwent ovariohysterectomy. Results are expressed as picomoles of KYNA/milliliter. The horizontal bar represents the median value.

[15]. The increase in KYNA concentration in the spinal cord has been demonstrated in the model of allergic encephalitis and myelitis in rats [16]. Elevated KYNA

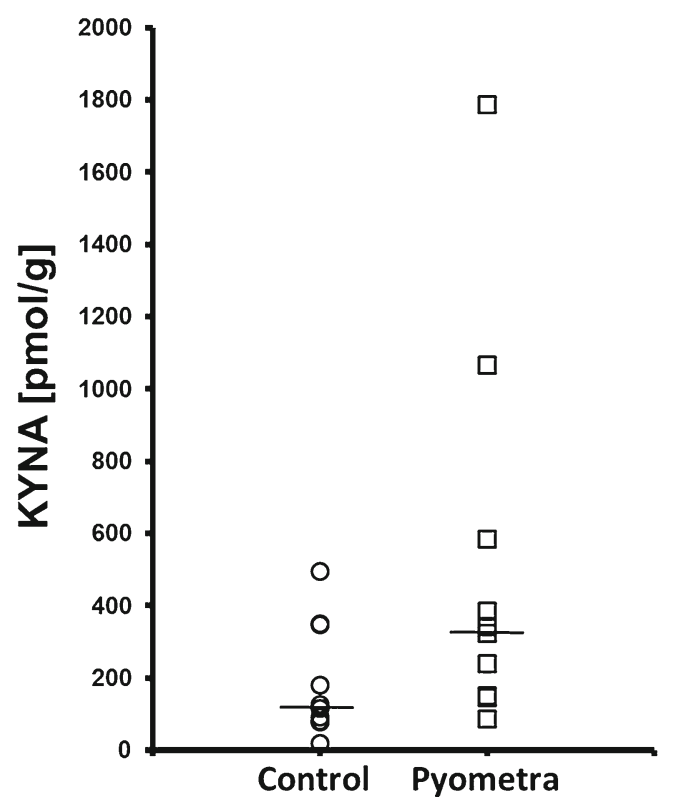

Fig. 2. Scatter plots of kynurenic acid (KYNA) concentration in endometrium of bitches. Samples were collected from bitches that underwent ovariohysterectomy. Results are expressed as picomoles of KYNA/gram wet weight. The horizontal bar represents the median value. concentration in the saliva of the patients with odontogenic abscesses has also been found [17]. In our study, a tendency toward an increase of KYNA concentration was found in the endometrium of bitches with pyometra; however, the difference did not reach statistical significance probably due to high variability between the results and limited number of investigated subjects.

It is known that frequency of pyometra increases with the age of animals [22]. In our study, the animals diagnosed with pyometra were significantly older than the control ones. It has been described that in 18-month old rats, the KYNA blood concentration is higher than in 3 -month old rats [23]. In contrast, no correlation between the KYNA blood concentration and age in humans has been observed [24]. We found no correlation between the age and KYNA blood concentration in bitches.

KYNA is produced from kynurenine, and the rate of its synthesis depends mainly on precursor availability. Kynurenine is converted from tryptophan by indoleamine 2,3-diooxygenase, which activity is stimulated in the course of inflammatory processes $[25,26]$. Moreover, it was found that lipopolysaccharide enhances KYNA production in human gingival fibroblasts [17]. Thus, it can be assumed that an increase in KYNA content is a result of inflammatory response.

Interestingly, data from animal studies indicate that an administration of exogenous KYNA results in antiinflammatory action. Glavin et al. demonstrated that KYNA attenuates experimental gastric ulcer formation produced by ethanol, stress, or toxins derived from the Atlantic coast mussels [27-29]. Kaszaki et al. found out that KYNA alleviates the inflammation resulting from experimental colon obstruction in dogs [30]. KYNA restored the tone of the colon and decreased motility index of the giant colonic contractions. It has been demonstrated that KYNA treatment significantly suppressed the increase in plasma nitrite/nitrate $\left(\mathrm{NO}_{\mathrm{x}}\right)$ and reduced elevated activity of xanthine oxidase and xanthine dehydrogenase [30]. Similarly, KYNA decreased the motility and increased the tone of the colon in the acute ulcerative colitis model. Moreover, it reduced elevated activity of xanthine oxidoreductase, nitric oxide synthase, and myeloperoxidase [31]. In septicemia induced by the administration of lipopolysaccharide in mice, KYNA significantly reduced the mortality rate of the animals and inhibited nitric oxide, as well as increased tumor necrosis factor $\alpha$ concentration [32].

Based on our results, a suggestion that KYNA concentration measurement might be an inflammation 
indicator of pyometra in bitches seems justified. It is also possible to call for application of KYNA in the treatment of pyometra, which requires confirmation in further experimental studies.

Open Access. This article is distributed under the terms of the Creative Commons Attribution License which permits any use, distribution, and reproduction in any medium, provided the original author(s) and the source are credited.

\section{REFERENCES}

1. Ellinger, A. 1904. Die entstehung der Kynurensaure. Zeitschrift für Physikalische Chemie 43: 325-337.

2. Okuno, E., F. Du, T. Ishikawa, M. Tsujimoto, M. Nakamura, R. Schwarcz, and R. Kido. 1990. Purification and characterization of kynurenine-pyruvate aminotransferase from rat kidney and brain. Brain Research 534: 37-44.

3. Birch, P.J., C.J. Grossman, and A.G. Hayes. 1988. Kynurenic acid antagonises responses to NMDA via an action at the strychnineinsensitive glycine receptor. European Journal of Pharmacology 154: 85-87.

4. Kessler, M., T. Terramani, G. Lynch, and M. Baudry. 1989. A glycine site associated with N-methyl-D-aspartic acid receptors: characterization and identification of a new class of antagonists. Journal of Neurochemistry 52: 1319-1328.

5. Wang, J., N. Simonavicius, X. Wu, G. Swaminath, J. Reagan, H. Tian, and L. Ling. 2006. Kynurenic acid as a ligand for orphan G protein-coupled receptor GPR35. Journal of Biological Chemistry 281: 22021-22028.

6. DiNatale, B.C., I.A. Murray, J.C. Schroeder, C.A. Flaveny, T.S. Lahoti, E.M. Laurenzana, C.J. Omiecinski, and G.H. Perdew. 2010. Kynurenic acid is a potent endogenous aryl hydrocarbon receptor ligand that synergistically induces interleukin-6 in the presence of inflammatory signaling. Toxicological Sciences 115: 89-97.

7. Zeden, J.P., G. Fusch, B. Holtfreter, J.C. Schefold, P. Reinke, G. Domanska, J.P. Haas, M. Gruendling, A. Westerholt, and C. Schuett. 2010. Excessive tryptophan catabolism along the kynurenine pathway precedes ongoing sepsis in critically ill patients. Anaesthesia and Intensive Care 38: 307-316.

8. Schefold, J.C., J.P. Zeden, C. Fotopoulou, S. von Haehling, R. Pschowski, D. Hasper, H.D. Volk, C. Schuett, and P. Reinke. 2009. Increased indoleamine 2,3-dioxygenase (IDO) activity and elevated serum levels of tryptophan catabolites in patients with chronic kidney disease: A possible link between chronic inflammation and uraemic symptoms. Nephrology, Dialysis, Transplantation 24: 1901-1908.

9. Heyes, M.P., K. Saito, J.S. Crowley, L.E. Davis, M.A. Demitrack, M. Der, L.A. Dilling, J. Elia, M.J. Kruesi, and A. Lackner. 1992. Quinolinic acid and kynurenine pathway metabolism in inflammatory and non-inflammatory neurological disease. Brain 115: 12491273.

10. Heyes, M.P., B.J. Brew, K. Saito, B.J. Quearry, R.W. Price, K. Lee, R.B. Bhalla, M. Der, and S.P. Markey. 1992. Interrelationships between quinolinic acid, neuroactive kynurenines, neopterin and beta 2-microglobulin in cerebrospinal fluid and serum of HIV-1-infected patients. Journal of Neuroimmunology 40: $71-80$.

11. Baran, H., J.A. Hainfellner, B. Kepplinger, P.R. Mazal, H. Schmid, and H. Budka. 2000. Kynurenic acid metabolism in the brain of HIV-1 infected patients. Journal of Neural Transmission 107: $1127-1138$.

12. Atlas, A., M. Gisslen, C. Nordin, L. Lindstrom, and L. Schwieler. 2007. Acute psychotic symptoms in HIV-1 infected patients are associated with increased levels of kynurenic acid in cerebrospinal fluid. Brain, Behavior, and Immunity 21: 86-91.

13. Heyes, M.P., E.K. Jordan, K. Lee, K. Saito, J.A. Frank, P.J. Snoy, S.P. Markey, and M. Gravell. 1992. Relationship of neurologic status in macaques infected with the simian immunodeficiency virus to cerebrospinal fluid quinolinic acid and kynurenic acid. Brain Research 570: 237-250.

14. Holtze, M., A. Mickiene, A. Atlas, L. Lindquist, and L. Schwieler. 2012. Elevated cerebrospinal fluid kynurenic acid levels in patients with tick-borne encephalitis. Journal of Internal Medicine. doi:10.1111/j.1365-2796.2012.02539.x.

15. Holtze, M., L. Asp, L. Schwieler, G. Engberg, and H. Karlsson. 2008. Induction of the kynurenine pathway by neurotropic influenza A virus infection. Journal of Neuroscience Research 86: 3674-3683.

16. Chiarugi, A., A. Cozzi, C. Ballerini, L. Massacesi, and F. Moroni. 2001. Kynurenine 3-mono-oxygenase activity and neurotoxic kynurenine metabolites increase in the spinal cord of rats with experimental allergic encephalomyelitis. Neuroscience 102: 687695.

17. Kuc, D., M. Rahnama, T. Tomaszewski, W. Rzeski, K. Wejksza, T. Urbanik-Sypniewska, J. Parada-Turska, M. Wielosz, and W.A. Turski. 2006. Kynurenic acid in human saliva - does it influence oral microflora? Pharmacological Reports 58: 393-398.

18. Hagman, R., H. Kindahl, and A.S. Lagerstedt. 2006. Pyometra in bitches induces elevated plasma endotoxin and prostaglandin F2alpha metabolite levels. Acta Veterinaria Scandinavica 47: 5567.

19. Nelson, R.W., and E.C. Feldman. 1986. Pyometra. The Veterinary Clinics of North America. Small Animal Practice 16: $561-576$.

20. Shibata, K. 1988. Fluorimetric micro-determination of kynurenic acid, an endogenous blocker of neurotoxicity, by high-performance liquid chromatography. Journal of Chromatography 430: 376-380.

21. Turski, W.A., M. Nakamura, W.P. Todd, B.K. Carpenter, W.O. Whetsell Jr., and R. Schwarcz. 1988. Identification and quantification of kynurenic acid in human brain tissue. Brain Research 454: 164-169.

22. Hardy, R.M., and C.A. Osborne. 1974. Canine pyometra: pathophysiology, diagnosis and treatment of uterine and extra-uterine lesions. Journal of the American Animal Hospital Association 10: $245-267$.

23. Moroni, F., P. Russi, V. Carla, and G. Lombardi. 1988. Kynurenic acid is present in the rat brain and its content increases during development and aging processes. Neuroscience Letters 94: 145150.

24. Ilzecka, J., T. Kocki, Z. Stelmasiak, and W.A. Turski. 2003. Endogenous protectant kynurenic acid in amyotrophic lateral sclerosis. Acta Neurologica Scandinavica 107: 412-418.

25. Prendergast, G.C., M.Y. Chang, L. Mandik-Nayak, R. Metz, and A.J. Muller. Indoleamine 2,3-dioxygenase as a modifier of pathogenic inflammation in cancer and other inflammation-associated diseases. Current Medicinal Chemistry 18: 2257-2262.

26. Boasso, A., and G.M. Shearer. How does indoleamine 2,3dioxygenase contribute to HIV-mediated immune dysregulation. Current Drug Metabolism 8: 217-223.

27. Glavin, G.B., and C. Pinsky. 1989. Kynurenic acid attenuates experimental ulcer formation and basal gastric acid secretion in 
rats. Research Communications in Chemical Pathology and Pharmacology 64: 111-119.

28. Glavin, G.B., R. Bose, and C. Pinsky. 1989. Kynurenic acid protects against gastroduodenal ulceration in mice injected with extracts from poisonous Atlantic shellfish. Progress in NeuroPsychopharmacology \& Biological Psychiatry 13: 569-572.

29. Glavin, G.B., C. Pinsky, and R. Bose. 1990. Gastrointestinal effects of contaminated mussels and putative antidotes thereof. Canada Diseases Weekly Report 16(Suppl 1E): 111-115.

30. Kaszaki, J., Z. Palasthy, D. Erczes, A. Racz, C. Torday, G. Varga, L. Vecsei, and M. Boros. 2008. Kynurenic acid inhibits intestinal hypermotility and xanthine oxidase activity during experimental colon obstruction in dogs. Neurogastroenterology and Motility 21: $53-62$.

31. Varga, G., D. Erces, B. Fazekas, M. Fulop, T. Kovacs, J. Kaszaki, F. Fulop, L. Vecsei, and M. Boros. 2010. N-MethylD-aspartate receptor antagonism decreases motility and inflammatory activation in the early phase of acute experimental colitis in the rat. Neurogastroenterology and Motility 22: 217225.

32. Moroni, F., A. Cozzi, M. Sili, and G. Mannaioni. 2012. Kynurenic acid: A metabolite with multiple actions and multiple targets in brain and periphery. Journal of Neural Transmission 119: 133139. 